\title{
Antimicrobial Resistance Profiles and Clonal Relatedness of Pseudomonas aeruginosa Strains Recovered from Wounds Infections of Outpatient Population Presenting in a Rural Hospital in Kenya
}

\author{
Thomas Gachuki Thuo $^{{ }^{*}}$ (D), Ciira Kiyuukia1, John Maina², Terry Judah², Susan Kiiru², John Kiiru² \\ ${ }^{1}$ Jomo Kenyatta University of Agriculture and Technology, Juja, Kenya \\ ${ }^{2}$ Kenya Medical Research Institute, Centre for Microbiology Research, Nairobi, Kenya \\ Email: *gacchukithomas@gmail.com
}

How to cite this paper: Thuo, T.G., Kiyuukia, C., Maina, J., Judah, T., Kiiru, S. and Kiiru, J. (2019) Antimicrobial Resistance Profiles and Clonal Relatedness of Pseudomonas aeruginosa Strains Recovered from Wounds Infections of Outpatient Population Presenting in a Rural Hospital in Kenya. Advances in Microbiology, 9, 119-130.

https://doi.org/10.4236/aim.2019.92009

Received: November 8, 2018

Accepted: January 27, 2019

Published: January 30, 2019

Copyright $\odot 2019$ by author(s) and Scientific Research Publishing Inc. This work is licensed under the Creative Commons Attribution International License (CC BY 4.0).

http://creativecommons.org/licenses/by/4.0/

\begin{abstract}
Pseudomonas aeruginosa is a leading cause of hospital infections and is intrinsically resistant to most antibiotics. Emergence of multidrug resistant (MDR) strains has been reported in the world and poses a great challenge in the management of infections associated with this species. While a substantial amount of research has been done on strains from most of other infection caused by this species in developed countries, little is known about the susceptibility profiles of strains recovered from African countries in general and Kenya in particular. Furthermore, there is paucity of data regarding strain, phenotype and genetic diversity of strains recoverable from wounds among patients in Kenya. The possible risk factors for acquisition of MDR strains and possible factors that could fuel clonal expansion in hospital and community settings remain undetermined. This cross-sectional study conducted in Tigoni Hospital, a rural area in Central Kenya sought to determine risk factors associated with carriage of MDR Pseudomonas aeruginosa in wounds among rural population. We also analyzed antimicrobial resistance profiles among these isolates. Prevalence of $P$. aeruginosa in wounds was $28 \%$ with 85 isolates recovered from wounds of 299 participants. Most antimicrobial resistance prevalence was recorded towards Ceftazidime (64\%) and Cefepime (52\%) while Piperacillin-tazobactam was the most effective antimicrobial agent with a resistance prevalence rate of $20 \%$. Resistance towards new classes of aminoglycosides such as Gentamicin was at $45 \%$ while that towards Amikacin was at $40 \%$. Compared to other related studies, relatively lower resistance towards Ciprofloxacin (25\%) and Meropenem (40\%) were recorded. Some
\end{abstract}


of the risk factors identified for carriages of MDR strains were self-medication (p: 0.001 , C.I: 3.01 - 8.86, O.R: 5.17) and non-completion of dosage (p: 0.12, C.I: 0.9 - 2.5, O.R: 1.5$)$.

\section{Keywords}

Carbapenems Resistance, Risk Factors Related to Carbepenems Resistance, Carriage of Pseudomonas aeruginosa in Wounds, Clonal Relatedness

\section{Introduction}

We detected bla $_{\mathrm{NDM}-1}$ in 10 isolates from a total of 34 isolates that were resistant to Meropenem. We were not able to determine genetic basis of resistance to this class of antimicrobials in the rest 24 isolates but these isolates have since been submitted for whole genome sequencing. $b a_{\text {TEM }}$ was the most prevalent $\beta$-lactamase gene at $25 \%$ while the prevalence of $b l a_{\mathrm{SHV}}$ was (24\%) was also recorded amongst strains resistant to $3^{\text {rd }}$ generation cephalosporins.

The phylogeny of recovered strains revealed significant genetic similarities among the strains. Our data revealed possible clonal expansion of some MDR strains. However tight clustering of strains that bore dissimilar resistance phenotypes further suggests independent acquisition of a similar set of resistance determinants among strains belonging to different clones. These isolates tested negative for other carbapenemases such as $b l a_{\mathrm{VIM}}$, or even KPC. This is the first report of carbapenemases resistant Pseudomonas from skin wounds in Kenya. The close relatedness phenotypically further indicates clonal expansion of these carbapenem resistant strains in the community. Unless something is done to curtail the dispersal of these strains, they could soon be implicated in large outbreaks in community and hospital settings thereby rising mortality and morbidity due to their insensitivity to majority of available drugs.

\section{Background}

Among the Pseudomonas species, Pseudomonas aeruginosa is the most important clinically. It is a Gram-negative rod ubiquitous in hospital environment especially in hot tubs, whirlpools, contact lens irrigation fluids, aerators, sinktraps, showers, and respiratory equipment. $P$. aeruginosa has been implicated in severe infections in immune-compromised individuals ranging from bacteremia, urinary tract infections, wound, respiratory, skin and burn infections. [1] [2] In the repertoire of infections caused by the organism wound infections come out as most common and therefore, the organism is a leading cause of life threatening wound infections often with high mortality rates [3]. This is in both chronic and acute wounds where several virulence factors come into play including the formation of biofilms. Factors such as loss of protection offered by intact epidermis through burns, puncture, wounds and eye trauma provide ideal condition for $P$. aeruginosa infections especially in hospital wards. Intrinsic resistance to multiple 
antibiotics in addition to formation of biofilms makes control and treatment of this organism difficult [4] [5]. This organism is able to thrive well in moist environment and therefore clonal spread of could cause of persistent nosocomial infections in the hospital environment. Indeed, previous studies in Kenya have documented $P$. aeruginosa to be the predominant cause of nosocomial infections in local hospitals [6]. In a study conducted in Agha Khan Hospital between 2006 and 2007, 57 Carbapenem resistant Pseudomonas aeruginosa isolates were obtained from blood, urine, respiratory tract and wound specimens. Notably $30 \%$ of these carbapenem resistant $P$ aeruginosa were obtained from wound specimens.

Antimicrobial resistance in $P$. aeruginosa has largely been attributed to production and acquisition of extended $\beta$-lactamases. These resistance mechanisms are mostly harbored in plasmid borne integron and are therefore easily transmissible across species and genera via horizontal gene transfer [7] [8]. Genetic elements such as Class 1 and 2 integron conferring resistance to $\beta$-lactams, aminoglycoside and fluoroquinolones have also been reported in $P$. aeruginosa. These classes of antimicrobials have high applicability in treatment of $P$. aeruginosa infections. Production and spread of carbapenemases that degrade carbapenems which are regarded as some of the drug of last resort for treating Gram-negative bacterial infections is on the rise among $P$. aeruginosa. This therefore makes treatment of associated infections more difficult and likely to result to treatment failure that could in turn result to prolonged hospitalization, high morbidity, and mortality.

Prevalence and antimicrobial resistance profiles of $P$. aeruginosa in rural Kenya have not been adequately studied. In urban centers, considerable self-medication and unhygienic environment have been among the major causes of antimicrobial resistance in such settings. In rural set-up on the other side, the drivers to this antimicrobial resistance have not been well documented. This cross-sectional study therefore set to determine prevalence of wound infections caused by $P$. aeruginosa and antimicrobial resistance in Tigoni hospital which is among tertiary hospital in Kiambu county attracting referrals within and beyond.

\section{Methodology}

\section{1) Study area and design}

A consecutive random sampling strategy was used in this cross-sectional study to obtain wound and swab specimen from consenting patients attending Tigoni District hospital for a period of six months in 2015. In total, 299 participants were recruited in this study where 299 of wound/swabs specimens were obtained. Of these specimens 240 (80\%) were from adults and 59 (20\%) were from children. Further, 183 (61\%) were obtained from males compared to 116 (39\%) from females. The mean age was 33.6 years and median age 29 years.

\section{2) Sample collection}

Patients with wounds were identified at the outpatient department, medical 
and surgical wards. A wound and/or pus swab obtained from the ailing participants was transported in Cary-Blair media in a cool box to the National Microbiology Reference Laboratory within three hours of collection. Unique codes were assigned to sample collection forms were transported along with the collected barcode samples. Social demographic data was captured included patient's age, gender, residence, antimicrobial source and ease in accessing over the counter, prescription availability and dose completion was captured on a structured questionnaire.

\section{3) Bacteria isolation and identification}

Pus and wound swabs were directly plated on sheep blood agar (SBA), Mueller Hinton agar and MacConkey with salt and incubated at $35^{\circ} \mathrm{C}$, for up to 24 hours. On MacConkey agar plate, non-lactose fermenters colonies with characteristic dry translucent edges were presumed as $P$. aeruginosa. On SBA, $P$. aeruginosa colonies were shiny, dry with rough edges, $\beta$-hemolytic with grape like smell. Identification was done using Gram stain and a series of biochemical tests such as Oxidase (positive), glucose fermentation, hydrolysis of arginine, and nitrate production test as previously described [9].

\section{4) Antimicrobial Susceptibility Testing}

Antimicrobial susceptibility tests were performed using Kirby-Bauer disc diffusion technique on Mueller Hinton agar (Oxoid). A McFarland turbidity standard of 0.5 was evenly spread on Mueller Hinton plate to obtain a confluent lawn. Antimicrobial discs included; Gentamicin (GEN $10 \mu \mathrm{g}$ ), Amikacin (AMK $30 \mu \mathrm{g}$ ), Ciprofloxacin (CIP $5 \mu \mathrm{g}$ ), Piperacillin-tazobactam (TZP 100/10 $\mu \mathrm{g}$ ), Ceftazidime (CAZ $30 \mu \mathrm{g})$, Cefepime (CPM $30 \mu \mathrm{g})$ and Meropenem (MEM $10 \mu \mathrm{g}$ ) were then dispensed. $P$. aeruginosa ATCC strains ATCC 27853 was used as control strain for media quality and disc potency. The plates were incubated overnight at $35^{\circ} \mathrm{C}$. The antimicrobial inhibition zone sizes were then measured and interpretation done based on the CLSI guidelines 2016.

\section{5) PCR screening of $\beta$-lactamase genes}

Isolates showing resistance to third generation cephalosporin were screening possible carriage of $b l a_{\mathrm{NDM}-1}, b l a_{\mathrm{SHV}}$ and $b l a_{\mathrm{TEM}}$ genes which have are the most reported variants in the region. Pure colonies sub-culture on Mueller Hinton agar were used for DNA extraction using the boiling method. Briefly, the method involved heating bacterial inoculums suspended in $1000 \mu \mathrm{l}$ of PCR water in an Eppendorf at $95^{\circ} \mathrm{C}$ for 12 minutes. Separation was then done by centrifugation at 14,000 rpm for 5 minutes. The supernatant containing separated DNA was template for the PCR process. Table 1 Finger print analysis using the (GTG) $)_{5}$ primer done to determine genetic relatedness of recovered Pseudomonas aeruginosa isolates. Amplified products were separated using 1.5 agarose gel and resultant bands visualized on gel max U.V imager.

\section{Results}

1) Analysis of risk factors in relation to Pseudomonas aeruginosa carriage amongst study participants 
Table 1. $\beta$-lactamase gene and finger printing primers.

\begin{tabular}{cccc}
\hline Gene & Primer sequence $\left(5^{\prime} \rightarrow 3^{\prime}\right)$ & Annealing $\left(\mathrm{T}^{\circ} \mathrm{C}\right)$ & Reference \\
\hline$(\mathrm{GTG})_{5}$ & GTGGTGGTGGTG & 40 & {$[10]$} \\
& F-TTATCTCCCTGTTAAGCCACC & 50 & {$[11]$} \\
& R-GATTTGCTGATTTCGCTCGG & & \\
$b a_{\mathrm{TEM}}$ & F-GCGGAACCCCTATTTG & 50 & {$[11]$} \\
& R-ACCAATGCTTAATCAGTGAG & & \\
$b a_{\mathrm{NDM}-1}$ & F-GAGATTGCCGAGCGACTTG & 61 & {$[12]$} \\
\hline
\end{tabular}

bla $a_{\mathrm{SHV}}$ : Sulhydryl $\beta$-lactamase variant, bla TEM $_{\mathrm{T}}$ : Temoneria $\beta$-lactamase variant, bla: $\beta$-lactamase gene, bla $a_{\mathrm{NDM}}$ : New Delhi $\beta$-lactamase, bp: molecular weight in base pair, F: forward primer, R: reverse primer.

A total of 85 non-duplicate $P$. aeruginosa strains were obtained from successful MacConkey and sheep blood cultures. Carriage of $P$. aeruginosa was high in wounds of adult (94\%) compared to those of children (6\%) with a significant statistical difference (P: 0.001, C.I: 2.1 - 14.0, O.R: 3.4). Similarly, wounds in females were more likely to be colonized at $67 \%$ compared to those of males at 33\% (P: 0.19, C.I: 0.84 - 2.4, O.R: 1.42).

Analysis of administered questionnaire further revealed that majority of the participants obtains antimicrobials from a community chemist as opposed to hospital pharmacy attributed to cost effectiveness. Carriage of $P$. aeruginosa was predominant among participants who obtained antimicrobial from community chemist (60\%) compared to those who acquired the same from hospital pharmacies $(40 \%)$ indicating that the source of antimicrobials determines the chances of colonization with this pathogen (P: 0.001, C.1: 3.01 - 8.86, O.R: 5.17).

Those who acquired antimicrobial without a doctor's prescription regardless of the source drug source were more likely to be colonized by a highly resistant strain compared to those to those had a prescription, P: 0.001, C.I: $3.01-8.86$, O.R: 5.17. Participant who recorded did not finish their antimicrobial dose had a higher carriage of these bacteria (50.6\%) compared to those who completed their dose (49.4\%) further indicating that adhering to dosage regimen is an important risk factor for colonization with this pathogen (P: 0.12, C.I: 0.9 - 2.5, O.R: 1.5).

2) Antimicrobial susceptibility patterns of Pseudomonas aeruginosa isolates

Among the antimicrobial tested, highest antimicrobial resistance was recorded towards Ceftazidime (64\%) whilst Piperacillin-tazobactam (80\%) was the most effective in-vitro (Figure 1). Gentamicin (45\%) was the least potent antimicrobial amongst the aminoglycoside tested. Pseudomonas aeruginosa resistance towards Ciprofloxacin (25\%) was least compared to compared to Cephalosporin and Aminoglycosides. High resistance towards Meropenem (40\%) was also recorded. More isolates from male participants were resistance to cephalosporins (CAZ 68\%, FEP 53\%) while those from females were more resistant to aminoglycoside (AK 80\%, CN 41\%). Bacteria isolates from participants aged between 


\section{Pseudomonas aeruginosa}

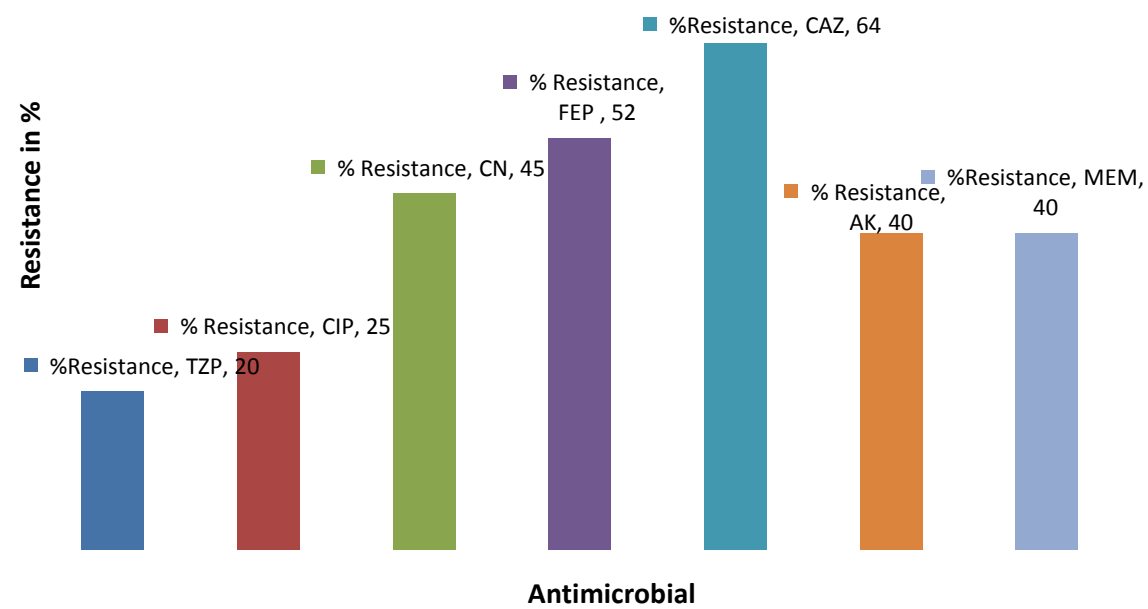

Figure 1. Antimicrobial resistance patterns of Pseudomonas aeruginosa isolates from wound samples. TZP: Tazobactum-piperacillin, CIP: Ciprofloxacin, CN: Gentamicin, FEP: Cefepime, CAZ: Ceftazidime, AK: Amikacin, MEM: Meropenem.

18 - 30 years were overall more resistant compared from those obtained from other age brackets (Table 2). A higher resistance towards virtually all tested antimicrobial agents was also recorded in bacterial isolates from participants who did not complete dosage compared to those that did.

\section{3) Carriage of b-lactamase genes}

bla $a_{\mathrm{NDM}-1}$ was detected in 10 isolates from a collection of 34 isolates that were resistant to Meropenem. These isolates were resistant to virtually all cephalosporins and aminoglycosides. The isolates were also highly resistant to and also to Tazobactam-Piperacillin and Ciprofloxacin (60\%). Carriage of TEM $\beta$-lactamase was the most predominant b-lactamase among the 34 isolates and in all isolates showing resistance to any b-lactam. Carriage of bla $a_{\mathrm{SHV}}$ was also recorded amongst Pseudomonas aeruginosa isolates resistant to Ceftazidime.

\section{4) Genetic relatedness of recovered Pseudomonas aeruginosa isolates}

High genetic similarity based on (GTG) [5] fingerprint was noted among Pseudomonas aeruginosa isolates from diverse patients visiting Tigoni Hospital Figure 2. A similarity of $\geq 80 \%$ was also noted in 9 clusters based on isolates banding patterns, participant area of residence, and source of antimicrobial agents, resistance phenotype and genotype. Several Pseudomonas aeruginosa isolates with identical resistance phenotypes were clustered together (cluster 3 ). Despite of significant genetic similarity of the 9 , most isolates had varying resistance phenotypes and genotype which perhaps is an indication that different clones have acquired these resistances independently. However the common factor among most isolates that clustered together was participant source of antimicrobial agent, either from private chemist or Hospital pharmacy. Notably the genetic basis of resistance (Table 3) in Pseudomonas aeruginosa isolates in the 9 cluster with significant genetic similarity was unknown, which suggest additional resistant mechanism from the targeted. 


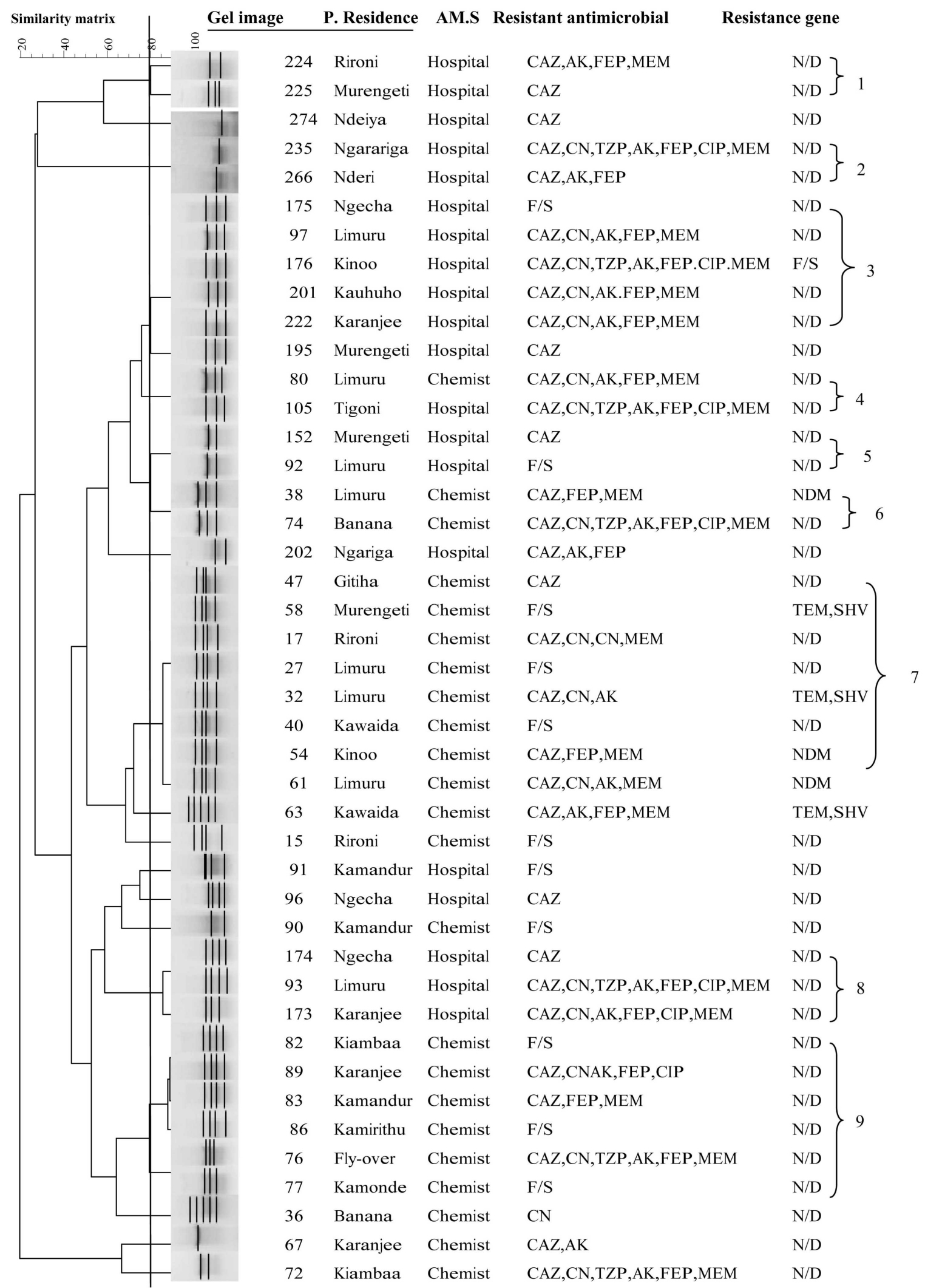

Figure 2. Fingerprint analysis of Pseudomonas aeruginosa isolates recovered from Patients visiting Tigoni Hospital. Key: P. residence: Participant's residence, AM.S: Source of antimicrobial, F/S: Fully susceptible, N/D: None of the target gene was detected. 
Table 2. Antimicrobial resistance patterns of $P$. aeruginosa isolates in relation to demographic features of the study paricipants.

\begin{tabular}{|c|c|c|c|c|c|c|c|c|}
\hline & \multicolumn{8}{|c|}{ Resistance \% } \\
\hline & $\mathbf{n}$ & $\mathrm{CN}$ & TZP & CIP & FEP & $\mathrm{CAZ}$ & $\mathrm{AK}$ & MEM \\
\hline All & 85 & 45 & 20 & 25 & 52 & 64 & 40 & 40 \\
\hline \multicolumn{9}{|l|}{ Gender of source } \\
\hline Males & 34 & 38 & 21 & 26 & 53 & 68 & 79 & 59 \\
\hline Females & 51 & 41 & 20 & 24 & 51 & 67 & 80 & 73 \\
\hline \multicolumn{9}{|l|}{ Age of source } \\
\hline Children $<13$ years & 5 & 20 & 0 & 0 & 60 & 80 & 100 & 80 \\
\hline Children $13-17$ & 3 & 33 & 0 & 0 & 0 & 0 & 100 & 0 \\
\hline Adults 18 - 30 & 35 & 51 & 34 & 37 & 66 & 83 & 83 & 83 \\
\hline Adults $31-50$ & 25 & 24 & 8 & 12 & 36 & 52 & 68 & 52 \\
\hline Adults $>50$ & 17 & 47 & 18 & 29 & 53 & 71 & 82 & 65 \\
\hline \multicolumn{9}{|l|}{ Prescription adherance } \\
\hline Dose completed & 4 & 0 & 0 & 0 & 75 & 100 & 75 & 75 \\
\hline Dose incompleted & 81 & 42 & 21 & 26 & 51 & 67 & 80 & 67 \\
\hline No prescription & 66 & 44 & 23 & 24 & 47 & 65 & 79 & 64 \\
\hline with prescription & 19 & 26 & 11 & 26 & 68 & 79 & 84 & 79 \\
\hline \multicolumn{9}{|l|}{ Source of drugs } \\
\hline Drugs from hospitals & 24 & 29 & 17 & 29 & 75 & 79 & 83 & 79 \\
\hline Drugs from chemist & 61 & 44 & 21 & 23 & 43 & 64 & 79 & 62 \\
\hline
\end{tabular}

TZP: Tazobactum-piperacillin, CIP: Ciprofloxacin, CN: Gentamicin, FEP: Cefepime, CAZ: Ceftazidime, AK: Amikacin, MEM: Meropenem, n: total count of corresponding feature.

Table 3. Resistant genes detected in Pseudomonas aeruginosa isolates.

\begin{tabular}{ccccccccc}
\hline \multirow{2}{*}{$\begin{array}{c}\text { Resistance gene } \\
\text { detected }\end{array}$} & Total detected & \multicolumn{7}{c}{ \% AMR in bla positive isolates } \\
\cline { 3 - 9 } & CAZ & CN & TZP & FEP & CIP & MEM & AK \\
\hline$b a_{\mathrm{TEM}}$ & 21 & 38 & 29 & 0 & 19 & 5 & 0 & 24 \\
bla $a_{\mathrm{SHV}}$ & 20 & 40 & 30 & 0 & 15 & 5 & 0 & 5 \\
$b l a_{\mathrm{NDM}}$ & 10 & 100 & 100 & 80 & 100 & 60 & 100 & 100 \\
\hline
\end{tabular}

bla $a_{\mathrm{NDM}}$ : New Delhi $\beta$-lactamase, bla $a_{\mathrm{SHV}}$ : Sulhydryl $\beta$-lactamase variant, $b l_{\mathrm{TEM}}$ : Temoneria $\beta$-lactamase variant, AMR: Antimicrobial resitance.

\section{Discussion}

In the current study, we recovered 85 Pseudomonas aeruginosa from wound swabs obtained from 299 outpatients visiting Tigoni Hospital. Clinical isolates of $P$. aeruginosa have previously been documented in Kenya. Such studies include a cross-sectional study conducted in Aga Khan University Hospital. The study reported $416 P$. aeruginosa urine, blood, wounds, purulent and respiratory tract specimens from patients admitted in Intensive care unit [6]. In this study, car- 
riage of Pseudomonas aeruginosa in wound specimen was 30\%, second to respiratory tract specimen (53\%).

Our findings showed that adults are at a higher risk of carriage Pseudomonas aeruginosa in wounds infections compared to those of children with a significant statistical difference (P: 0.001, C.I: 2.1 - 14.0, O.R: 3.4). Similarly, female were more likely to be colonized at $67 \%$ carriage compared to those of males at $33 \%$ (P: 0.19 , C.I: $0.84-2.4$, O.R: 1.42). Further analysis also revealed that most individuals opted to obtain medication from community chemists as opposed to hospital pharmacies with a significant difference in carriage of $P$. aeruginosa in wound infections been noted (P: 0.001, C.1: 3.01 - 8.86, O.R: 5.17). Although we did not go a further notch to determine the possible cause of the above difference, it is very likely that some of the medicine available in the community chemist are counterfeits therefore not effective in clearing infections. Self-medication is a major public health problem and possible driver of antimicrobial resistance in the country. Miss-use of antimicrobial agents was not an exception among study participants where the menace was significantly associated with Pseudomonas aeruginosa carriage (P: 0.001, C.1: $3.01-8.86$, O.R: 5.17). It was also noted that most of the self-medicating did not have disease diagnosis and therefore medication was non-specific and often not in full dosage. It was further noted that majority of self-medicating individuals did not have a doctor's prescription. Poverty and high doctor's consultation fee were noted as major cause of self-medication preference among this population.

In addition to the intrinsic antimicrobial resistance in Pseudomonas aeruginosa, misuse and over use of antimicrobial agents have led to emergence of multiple resistance strains that are difficult to treat. In addition, $P$. aeruginosa have become resistance to antimicrobial agents through horizontal acquisition of resistance genes from resistant strains and other bacterial species such Acinetobacter baumannii. Findings of the current study indicate an overall resistance to test antimicrobial agents compared to a study conducted in Kenyatta National hospital that recorded high resistance to CAZ 70\%, AK 46\%, CN 67.9\%, CIP $52.7 \%$, TZP 50.5\% and MEM 67.6\% [13] In this study were recorded high resistance prevalence to important classes of antibiotics such as CAZ (64\%), Gentamicin at $45 \%$ and Ciprofloxacin at, $25 \%$. A combination of these antimicrobials is used in the treatment of severe $P$. aeruginosa infections and therefore, resistance to a combination of such agents may greatly hamper treatment. Resistance to carbapenem which is the last resort was also recorded (MEM, 40\%). Our findings therefore show an increase in carbapenem resistance in East Africa as compared to a systematic review done in the region that reported an incidence of $21 \%$ [14]. Tazobactam-Piperacillin the most used anti-Pseudomonal agent was effective to most isolates that were resistant to other antimicrobials

Resistance to third generation $\beta$-lactams such as CAZ has been attributed to carriage of $\beta$-lactamases [15] bla $a_{\mathrm{NDM}}$ carriage was positive in only 10 from a total of 34 isolates that were resistant to Meropenem. However, only $40 \%$ of these 
isolates with this gene were positive for $b a_{\mathrm{TEM}}$ or $b a_{\mathrm{SHV}}$ indicating that there is little evidence of co-carriage of this gene with other bla genes as described in a recent study on $P$. aeruginosa strains from Kenyatta hospital wards in Kenya [13]. The above mentioned study also recorded 51.9\% bla $a_{\mathrm{NDM}}$ and $49.6 \%$. bla $a_{\mathrm{VEB}}$ carriage among 188 Pseudomonas aeruginosa isolates from urine, blood, respiratory tract and pus specimen. This may therefore infer possible carriage of other $\beta$-lactamase genes in our Pseudomonas aeruginosa isolates [3]. However, the DNA extracts of these bacterial isolates have since been sent for whole genome sequencing which further help in determine their genetic structure. Some meropenem isolates tested negative for the other carbapenems and these have been submitted WGS. We recorded high resistances to $\beta$-lactams including carbapenems. It is significant that these isolates were obtained from people who were not necessarily treating wounds. Since some of the wounds were open, there is a possibility that such strains may be coming from the environment especially soil.

The phylogeny of recovered strains revealed significant genetic similarity among our bacterial isolates. Further analysis also showed possible clonal expansion of resistant strains within the hospital that has identical resistance phenotype and a high genetic similarity. Tight clustering of isolates with different resistance phenotypes further suggests independent acquisition of a similar set of resistance determinants among isolates with different profiles. Although we were not able to determine possible carriage of genetic elements such as plasmids that could possible cause above mention resistance features, further genetic analysis and SNP typing based on WGS data will shed light into this. Although (GTG) 5 is not a very powerful phylogeny method, there is possibility that the NDM strains are clonal going by their clustering and resistance profiles. It is worth noting that the non-NDM strains did not cluster tightly which raises the possibility of clone stability of the highly resistant NDM strains.

The spread of clonal strains in clinical set-up has been associated with use medical devices such as catheters and prosthetics [16]. Pseudomonas aeruginosa strains are able to survive in the environment especially in most areas. Therefore, person to person transmission is also possible especially in contaminated environment. There should therefore be deliberate effort to reduce transmission and source reservoir of such resistance strains in such settings.

\section{Conclusion}

The emergence of $b l a_{\mathrm{NDM}-1}$ linked to widespread misuse of carbapenem is alarming. Measures to institutionalize antimicrobial stewardship such as hospital committees, prescription guided by empirical laboratory susceptibility patterns and continued monitoring of development of resistance should therefore be initiated. Risk factor associated with carriage and acquisition of multiple-drug resistance strains such as self-medication should therefore be strongly discouraged. Measures to closely monitor dosage completion and post-treatment follow-up for serious infections should also be initiated where possible to prevent 
emergence of antimicrobial resistance.

\section{Conflicts of Interest}

The authors declare no conflicts of interest regarding the publication of this paper.

\section{References}

[1] Ahmed, S.H., Daef, E.A., Badary, M.S., Mahmoud, M.A. and Abd-Elsayed, A.A. (2009) Nosocomial Blood Stream Infection in Intensive Care Units at Assiut University Hospitals (Upper Egypt) with Special Reference to Extended Spectrum Beta-Lactamase Producing Organisms. BMC Research Notes, 2, 76. https://doi.org/10.1186/1756-0500-2-76

[2] Sanchez, A., Gattarello, S. and Rello, J. (2011) New Treatment Options for Infections Caused by Multiresistant Strains of Pseudomonas aeruginosa and Other Nonfermenting Gram-Negative Bacilli. Seminars in Respiratory and Critical Care Medicine, 32, 151-158. https://doi.org/10.1055/s-0031-1275527

[3] Farshadzadeh, Z., Khosravi, A.D., Alavi, S.M., Parhizgari, N. and Hoveizavi, H. (2014) Spread of Extended-Spectrum Beta-Lactamase Genes of blaOXA-10, blaPER-1 and blaCTX-M in Pseudomonas aeruginosa Strains Isolated from Burn Patients 1. Burns, 40, 1575-1580. https://doi.org/10.1016/j.burns.2014.02.008

[4] Magiorakos, A.P., Srinivasan, A., Carey, R.B., et al. (2012) Multidrug-Resistant, Extensively Drug-Resistant and Pandrug-Resistant Bacteria: An International Expert Proposal for Interim Standard Definitions for Acquired Resistance. Clinical Microbiology and Infection, 18, 268-281.

https://doi.org/10.1111/j.1469-0691.2011.03570.x

[5] Oli, A.N., Eze, D.E., Gugu, T.H., Ezeobi, I., Maduagwu, U.N. and Ihekwereme, C.P. (2017) Multi-Antibiotic Resistant Extended-Spectrum Beta-Lactamase Producing Bacteria Pose a Challenge to the Effective Treatment of Wound and Skin Infections. Pan African Medical Journal, 27, 66. https://doi.org/10.11604/pamj.2017.27.66.10226

[6] Pitout, J.D., Revathi, G., Chow, B.L., et al. (2008) Metallo-Beta-Lactamase-Producing Pseudomonas aeruginosa Isolated from a Large Tertiary Centre in Kenya 1. Clinical Microbiology and Infection, 14, 755-759. https://doi.org/10.1111/j.1469-0691.2008.02030.x

[7] Jeong, J.H., Shin, K.S., Lee, J.W., Park, E.J. and Son, S.Y. (2009) Analysis of a Novel Class 1 Integron Containing Metallo-Beta-Lactamase Gene VIM-2 in Pseudomonas aeruginosa. Journal of Microbiology, 47, 753-759.

https://doi.org/10.1007/s12275-008-0272-2

[8] Woodford, N., Zhang, J., Kaufmann, M.E., et al. (2008) Detection of Pseudomonas aeruginosa Isolates Producing VEB-Type Extended-Spectrum Beta-Lactamases in the United Kingdom 1. Journal of Antimicrobial Chemotherapy, 62, 1265-1268. https://doi.org/10.1093/jac/dkn400

[9] Al-Charrakh, A.H., Al-Awadi, S.J. and Mohammed, A.S. (2016) Detection of Metallo-Beta-Lactamase Producing Pseudomonas aeruginosa Isolated from Public and Private Hospitals in Baghdad, Iraq. Acta Medica Iranica, 54, 107-113.

[10] Mohapatra, B.R., Broersma, K. and Mazumder, A. (2007) Comparison of Five rep-PCR Genomic Fingerprinting Methods for Differentiation of Fecal Escherichia coli from Humans, Poultry and Wild Birds. FEMS Microbiology Letters, 277, 
98-106. https://doi.org/10.1111/j.1574-6968.2007.00948.x

[11] Hasman, H., Mevius, D., Veldman, K., Olesen, I. and Aarestrup, F.M. (2005) Beta-Lactamases among Extended-Spectrum Beta-Lactamase (ESBL)-Resistant Salmonella from Poultry, Poultry Products and Human Patients in The Netherlands. Journal of Antimicrobial Chemotherapy, 56, 115-121.

https://doi.org/10.1093/jac/dki190

[12] Teo, J.W., La, M.V., Jureen, R. and Lin, R.T. (2015) Emergence of a New Delhi Metallo-Beta-Lactamase-1-Producing Pseudomonas aeruginosa in Singapore 1. Emerging Microbes \& Infections, 4, e72. https://doi.org/10.1038/emi.2015.72

[13] Mukaya, K.J.S., Maina, J., Museve, B., Nyerere, A.K. and Kiiru, J. (2018) Antimicrobial Resistance Profile and Genetic Profiling of Pseudomonas aeruginosa Strains Obtained from Different Inpatient Wards at Kenyatta National Hospital. IOSR Journal of Pharmacy and Biological Sciences (IOSR-JPBS), 13

[14] Ssekatawa, K., Byarugaba, D.K., Wampande, E. and Ejobi, F. (2018) A Systematic Review: The Current Status of Carbapenem Resistance in East Africa. BMC Research Notes, 11, 629. https://doi.org/10.1186/s13104-018-3738-2

[15] Bush, K. and Jacoby, G.A. (2010) Updated Functional Classification of Beta-Lactamases. Antimicrobial Agents and Chemotherapy, 54, 969-976.

https://doi.org/10.1128/AAC.01009-09

[16] Aiken, A.M., Mturi, N., Njuguna, P., et al. (2011) Risk and Causes of Paediatric Hospital-Acquired Bacteraemia in Kilifi District Hospital, Kenya: A Prospective Cohort Study. Lancet, 378, 2021-2027.

https://doi.org/10.1016/S0140-6736(11)61622-X 